\title{
El arbitraje en línea en la resolución de controversias por nombres de dominio punto $\mathrm{cl}$
}

\author{
Online Arbitration in Dispute Resolution \\ of Dot CL Domain Names
}

LUIS ARANCIBIA MEDINA

Coordinador Centro de Resolución de Controversias, NIC Chile

PATRICIO V. POBLETE

Director, NIC Chile. Profesor Titular, Universidad de Chile

JOSÉ URZÚA REINOSO

Jefe de Desarrollo de Sistemas, NIC Chile

MARGARITA VALDÉS CORTÉS

Directora Legal y Comercial, NIC Chile

RESUMEN Este artículo proporciona una explicación detallada sobre el procedimiento de arbitraje en línea para la resolución de disputas de nombres de dominio punto cl. Asimismo, describe los antecedentes y las etapas hacia el desarrollo e implementación del proyecto. También proporciona estadísticas sobre este nuevo modelo de arbitraje electrónico, cuyo establecimiento se ha convertido en una experiencia de innovación significativa en el derecho procesal chileno.

PALABRAS CLAVE Nombres de dominio punto cl, resolución de controversias, arbitraje electrónico. 
ABSTRACT This paper presents in detail the procedure for online arbitration for domain name dispute resolution in the dot $\mathrm{cl}$ top level domain. It also describes the previous situation and the stages of the development and implementation of the project. It also includes statistics about this new model of electronic arbitration that has become a significant innovation in Chilean procedural law.

KEYWORDS Dot cl domain names, dispute resolution, electronic arbitration.

\section{INTRODUCCIÓN}

Desde el año I986, la administración del Registro de Nombres del Dominio CL, denominado Network Information Center Chile (NIC Chile), está gestionada por el Departamento de Ciencias de la Computación de la Universidad de Chile por delegación de la Internet Assigned Numbers Authority (IANA), ${ }^{\mathrm{I}}$ de acuerdo a los principios contenidos en RFC I 59 I: Domain Name System Structure and Delegation. ${ }^{2}$ Dicha delegación fue reconocida formalmente el 24 de junio de 2006 mediante un acuerdo

I. Relacionado con IANA, el día I de octubre de 2016 concluyó un proceso prolongado y de gran importancia para el desarrollo de la red mundial. Desde sus inicios, y especialmente desde fines de los años noventa, el gobierno de Estados Unidos había tenido una posición privilegiada en la supervisión de algunos servicios críticos de internet. En particular, todos los cambios en el nivel superior del sistema de nombres de dominio (DNS) debían ser autorizados por dicho gobierno, a través de su Departamento de Comercio y, más específicamente, por la National Telecommunications and Information Agency (NTIA). En principio, esto le daba poder para vetar la creación de nuevos dominios o incluso para suspender o hasta eliminar dominios existentes, tales como los punto com o punto cl. En la práctica, dicho poder nunca se ejerció y esta supervisión siempre se realizó como un trámite más dentro de la rutina del funcionamiento de esos servicios, los cuales son realizados como una función llamada Internet Assigned Numbers Authority (IANA), la cual a su vez está a cargo de la Internet Corporation for Assigned Names and Numbers, ICANN. El gran cambio producido a partir de esta fecha es que Estados Unidos ha renunciado voluntariamente a este poder sobre la IANA, transfiriendo esta responsabilidad a ICANN, quien la ejercerá supervisada por la comunidad Internet global, a través de diversos mecanismos establecidos para este efecto. Al respecto, véase Valdés (20I6) y Poblete (20I6).

2. Véase http://www.ietf.org/rfc/rfc I 59 I.txt. 
marco suscrito entre ICANN y NIC Chile por el cual se estableció las responsabilidades que ambas entidades tienen en la preservación de la estabilidad, la seguridad y la interoperabilidad de internet. ${ }^{3}$

Desde los inicios de la formalización del servicio de registro y administración del sistema de nombres de dominio punto cl, el arbitraje ha sido el método empleado para resolver disputas. Para comprender adecuadamente las razones que han derivado en el diseño y establecimiento de un sistema de arbitraje en línea de resolución de controversias, es necesario revisar previamente el itinerario de los principales eventos del devenir del primer diseño y las versiones posteriores del procedimiento arbitral que rigió hasta diciembre de 2013. El tránsito a un sistema electrónico supuso la decantación de aprendizajes que terminaron por impulsar el desarrollo de un modelamiento que fuera funcional para el ejercicio de la jurisdicción arbitral, junto con implementar una herramienta segura de tutela de los derechos de los litigantes.

El hito fundacional del camino seguido se produjo el 9 septiembre de 1997 cuando entró en vigencia la primera versión de la denominada «Reglamentación para el Funcionamiento del Registro de Nombres del Dominio CL». En dicho conjunto de normas, junto con establecerse reglas para la prestación de los servicios de registro y administración para los nombres de dominio punto $\mathrm{cl}{ }^{4}$ se decidió que el arbitraje sería el método de resolución de controversias. A través de dicho modelamiento, que en lo fundamental estuvo en operaciones hasta el I de diciembre de 20I3, NIC Chile se transformó en una institución pionera en el mundo en el uso de arbitraje para resolver este tipo de conflictos. Muchos fueron los factores de la adopción del arbitraje y su posterior desarrollo exitoso, entre otros: la existencia de una cultura legal favorable y el hecho de que se tratara de una herramienta adecuada para proveer una solución rápida y de bajo costo de los litigios, ${ }^{5}$ que además dotaba de resguardo

3. Véase https://www.nic.cl/acerca/documentos/ICANN-Accountability.pdf.

4. Éste fue el primer conjunto explícito de normas para el servicio de registro y administración de nombres de dominio punto cl, que estableció el cobro de una tarifa por la inscripción de los dominios y fue el inicio del desarrollo de una práctica de registro basada en información de los titulares de dominio estandarizada y de acceso público.

5. El factor de los costos del arbitraje ha sido tratado, con especial referencia al caso del sistema administrado por NIC Chile en (Carey y Fernández, 2007: 21 I y ss). 
jurídico a la resolución de los conflictos a través de un mecanismo legal y constitucionalmente reconocido.

El rol de NIC Chile como entidad administradora del sistema de resolución de controversias quedó establecido desde los inicios del desarrollo del modelo de resolución de controversias. Las principales funciones en este sentido han sido aquellas vinculadas exclusivamente con la provisión de servicios de apoyo a la gestión resolutiva de disputas, de manera independiente y con total prescindencia de la cuestión discutida en el juicio arbitral. Tales actividades de soporte han sido, principalmente, el nombramiento del árbitro por sorteo, en caso que los solicitantes hubieran omitido designar uno de común acuerdo, y el cumplimiento de la sentencia luego de la notificación arbitral.

En aquella primera reglamentación se estableció que toda solicitud de inscripción de un dominio se publicaba en el sitio web www.nic.cl por 30 días, período en el que se admitían otras solicitudes competitivas para que cualquier interesado pudiera invocar algún derecho preexistente respecto del mismo nombre. En caso de que así ocurriera, se daba inicio al arbitraje entre las solicitudes presentadas. Si no había otra solicitud en trámite para el nombre solicitado, el dominio era asignado al vencimiento de dicho plazo de 30 días. ${ }^{6}$

Con la finalidad de perfeccionar el modelo y atendiendo a la experiencia obtenida en los primeros años del sistema, hacia finales del año I999 se hicieron importantes cambios en el procedimiento de resolución de disputas. Por un lado, se introdujo la denominada acción de revocación ${ }^{7}$ con la que se construyó un nuevo supuesto para la disputa de

\footnotetext{
6. Una evaluación de los primeros resultados del arbitraje se puede encontrar en Piquer y Poblete (I999).

7. El antecedente inmediato y directo de la acción de revocación es la acción contenida en la Uniform Domain-Name Dispute-Resolution Policy (UDRP), adoptada en la reunión ICANN de Santiago de Chile, el 26 de agosto de I999. La acción de revocación se introdujo en el artículo 22 de aquella primera Reglamentación. En dicha disposición se estableció que: «Será causal de revocación de un nombre de dominio el que su inscripción sea abusiva, o que ella haya sido realizada de mala fe. La inscripción de un nombre de dominio se considerará abusiva cuando se cumplan las tres condiciones siguientes: i) que el nombre de dominio sea idéntico o engañosamente similar a una marca de producto o de servicio sobre la que tiene derechos el reclamante, o a un nombre por el cual el reclamante es reconocido; ii) que el asignatario del nombre de dominio no tenga dere-
} 
un dominio, dado que fue posible controvertir un dominio inscrito por cualquier titular de derechos marcarios y también cualquier persona que pudiera invocar otro derecho sobre el nombre asignado. ${ }^{8}$

La otra modificación del año I999 fue el establecimiento de la mediación, en virtud de la cual los solicitantes en competencia por el dominio o en proceso de revocación eran citados a una audiencia ante un mediador experto de NIC Chile, en función de la búsqueda de un acuerdo que pusiera fin a la disputa. En caso de no existir un acuerdo entre ellos, el asunto era derivado al conocimiento de un árbitro. La mediación abrió un espacio institucionalizado para el encuentro entre los usuarios en conflicto y constituyó una novedad, en el contexto de la masificación de los mecanismos alternativos de resolución de disputas que empezaban a instalarse en distintos ámbitos del derecho en nuestro país, a la par que se consolidó como un mecanismo que aportó de manera significativa a educar a los usuarios y a resolver disputas por nombres de dominio.

A partir del año 2000 se produjo un rápido crecimiento de internet en nuestro país. Tal escenario estimuló el aumento importante de las solicitudes de dominios punto $\mathrm{cl},{ }^{9}$ lo cual trajo como efecto el aumento en la cantidad de conflictos que el sistema tuvo que administrar, buscando ofrecer a los usuarios un mecanismo de defensa frente a prácticas como la ciberocupación, pero al mismo tiempo tratando de desincentivar prácticas de signo opuesto, como el reverse domain name hijacking. ${ }^{10}$

chos o intereses legítimos con respecto del nombre de dominio; y iii) que el nombre de dominio haya sido inscrito y se utilice de mala fe.

8. La primera versión de la tramitación de la acción de revocación contemplaba un «examen de admisibilidad» que buscaba evaluar preliminarmente la pertinencia de la solicitud para evitar que el sistema pudiera dar lugar a abusos por parte de los usuarios. Sin embargo, en $200 \mathrm{I}$, tras dos años desde que esa medida fuera implementada, se pudo constatar que las disputas eran fundadas y no se trataba de acciones temerarias, por lo que dicho examen se eliminó. En la misma ocasión, se eliminó el plazo de tres años señalado inicialmente para el ejercicio de la acción. Cabe agregar que durante la vigencia de la Reglamentación de I997, modificada en I999, los conflictos por revocación representaron una minoría en el total de los conflictos administrados por el sistema. Esto implicó que, del total de disputas resueltas en el Sistema de Resolución de Disputas, los procedimientos por revocación tuvieron una baja incidencia.

9. Véase el crecimiento del stock de dominios inscritos en http://www.nic.cl/estadisticas/crecimiento-detalle.html.

ıo. Ciberocupación es un término utilizado para describir el registro abusivo, delibe- 
Dado que el plantel de árbitros existente hasta esa fecha era insuficiente para manejar la creciente carga de trabajo, se hizo necesario aumentar la dotación de abogados habilitados para hacer frente a la situación. El procedimiento que se utilizó para la selección de dichos profesionales fue el concurso público. ${ }^{\text {II }}$

\section{CARACTERÍSTICAS DEL MECANISMO DE RESOLUCIÓN DE CONTROVERSIAS VIGENTE HASTA EL 1 DE DICIEMBRE DE 2013}

El Sistema de Resolución de Controversias vigente hasta la fecha señalada estaba construido sobre las siguientes bases:

- Un procedimiento bifásico conformado por una audiencia de mediación, como etapa previa al arbitraje.

- La audiencia de mediación era un servicio gratuito para los usuarios en controversia y de asistencia voluntaria.

- A falta de acuerdo entre los solicitantes en conflicto, el asunto debía solucionarse por un arbitraje de árbitro arbitrador.

- Las bases del procedimiento arbitral eran acordadas entre el árbitro y las partes. ${ }^{\mathrm{I} 2}$

- El arbitraje era presencial, con un uso reducido de medios electró-

rado y de mala fe de un nombre de dominio en violación a los derechos de marcas de producto o servicio (véase el documento de la OMPI disponible en http://www.wipo.int/ amc/es/processes/processi/report/finalreport.html). Reverse Domain Name Hijacking o secuestro inverso de nombres de dominio consiste en el uso de la Política de Resolución de Controversias de manera abusiva para intentar privar a un titular de nombre de dominio que ha registrado de buena fe. Véase el párrafo I (Definiciones) del Reglamento para una Política Uniforme de Resolución de Disputas sobre Nombres de Dominio, OMPI, disponible en http://www.wipo.int/amc/es/domains/gtld/.

I I. Durante la Reglamentación vigente hasta el I de diciembre de 20 I 3 se convocó a dos concursos de selección, con lo que el grupo de árbitros del Sistema de Resolución de Controversias de NIC Chile alcanzó un total de 23 abogados habilitados para resolver las disputas.

I2. Aunque fue decantando un conjunto de reglas comunes entre todos los árbitros, la determinación precisa de las normas de tramitación era de exclusiva incumbencia del árbitro y las partes. 
nicos para la gestión del juicio. ${ }^{\mathrm{I3}}$

- El monto de los honorarios arbitrales era fijado por el árbitro y la parte obligada a su pago era el demandante. ${ }^{\mathrm{I}}$

- Árbitros seleccionados mediante concurso público e independientes de NIC Chile o de la Universidad de Chile. Como jueces que son, están sujetos a la superintendencia directiva, correccional y económica de la Corte Suprema de Justicia.

\section{LA NUEVA REGLAMENTACIÓN Y SUS POLÍTICAS}

El I de diciembre de 2013 entró en vigencia la nueva reglamentación, ${ }^{\mathrm{I5}}$ junto a nuevas políticas y procedimientos enfocados todos ellos a adecuarse a las prácticas generalmente aceptadas por la industria de los nombres de dominio, especialmente en lo relativo a la inscripción y gestión de un nombre de dominio y a la adopción del modelo registry/registrar para los punto cl. ${ }^{16}$

Las nuevas políticas introdujeron cambios significativos en el esque-

I3. A partir del 20 de septiembre 2005 se adoptó una serie de correctivos orientados a la agilización de la tramitación de los expedientes en el Sistema de Administración de Controversias. Entre estos cambios se encontraba la reducción del plazo para efectuar las tachas al listado de árbitros, el que sería de 5 días hábiles. Asimismo, se introdujo el uso intensivo de la firma electrónica en las comunicaciones entre la Administración del Sistema de Solución de Controversias y los árbitros, entre otros cambios. Véase http:// www.nic.cl/anuncios/20050920-reglamentacion.html.

I4. El 5 de septiembre de 200I, siguiendo las recomendaciones del Grupo de Trabajo sobre Nombres de Dominio que se había establecido a finales del año 2000 con la Subsecretaría de Economía, se liberó del pago de los honorarios del arbitraje al primer solicitante o al titular del dominio, en las disputas por inscripción o revocaciones, respectivamente.

I 5. Véase http://www.nic.cl/normativa/reglamentacion.html.

I6. El cambio de lo que se denominó el Nuevo CL estuvo articulado en varias normas, políticas y procedimientos. Junto a la Nueva Reglamentación, entró en vigencia el Procedimiento sobre Cuentas de Usuario (véase http://www.nic.cl/normativa/procedimientocuentas-usuario.html) y la Política de Resolución de Controversias. El diseño de las nuevas normas fue consecuencia de un proceso de consultas y de trabajo en conjunto con la comunidad representada en el Consejo Nacional de Nombres de Dominio y Números IP el que recomendó a NIC Chile el I 4 de octubre de 20 i I la adopción de la Nueva Reglamentación y su Política de Resolución de Controversias. 
ma del servicio de inscripción y administración de los nombres de dominio punto $\mathrm{cl}$, dentro de los que se puede mencionar:

- La inscripción inmediata de un nombre de dominio una vez recibido el pago, eliminando la categoría de «solicitudes en trámite».

- La exigencia de una cuenta de usuario para inscribir un nombre de dominio, la cual es la herramienta donde los usuarios pueden administrar todos los procesos relacionados con sus registros: renovación, cambios de titular, modificación de contactos, etcétera.

- La admisión de la inscripción directa de nombres de dominio por parte de extranjeros, sin necesidad de requerir de un representante local.

- La adecuación de la normativa y de los sistemas para permitir la existencia agentes registradores de punto $\mathrm{cl}$ (registrars). ${ }^{\mathrm{I7}}$

\section{LA POLÍTICA DE RESOLUCIÓN DE CONTROVERSIAS POR NOMBRES DE DOMINIO CL ${ }^{18}$}

Una de las piezas que construyen la arquitectura de la nueva reglamentación es la Política de Resolución de Controversias por Nombres de Dominio CL. Con la vigencia de dicha política se introdujo el procedimiento de arbitraje en línea como un cambio trascendental en la manera de disputar y administrar los conflictos por nombres de dominio. En general, dicho procedimiento es una construcción que incorpora los conocimientos, la experiencia y los aprendizajes adquiridos desde el primer modelo de arbitraje y los requerimientos de la comunidad relevante.

Se trata, en consecuencia, de un diseño estructurado en base a la práctica y a las habilidades adquiridas en la gestión administrativa del Sistema de Resolución de Controversias, todas las cuales, combinadas con el uso de diferentes aplicaciones informáticas, han sido puestas al servicio

I7. La Política de Acreditación de Agentes Registradores para Dominios CL ha comenzado a operar recientemente bajo la forma de un plan piloto. Véase http://www. nic.cl/anuncios/20160909-registrars.html. Los agentes registradores son entidades acreditadas a través de un procedimiento por el cual quedan habilitadas para comercializar nombres de dominio y realizar todas las operaciones necesarias para su gestión.

I 8. Véase http://www.nic.cl/normativa/politica-RCAL.html. 
de la tramitación y litigación remota de los conflictos por nombres de dominio. A lo anterior se agrega la conformación de un equipo multidisciplinario, compuesto principalmente por ingenieros y abogados, lo que permitió un trabajo en conjunto entre las áreas de Ingeniería, Legal y Expertos externos. Con esto, se logró una interacción fluida, permitiendo abordar la implementación y pruebas de las funcionalidades considerando diferentes puntos de vista.

Con el transcurso del tiempo se pudo constatar que era necesario introducir cambios en el procedimiento de resolución de controversias. Una de las razones era que el modelo seguido hasta la fecha de la entrada en vigencia de las nuevas políticas no era concordante con la necesidad de facilitar el acceso a estos procedimientos a todos los usuarios, desde cualquier lugar de Chile, ni con las exigencias de una verdadera «internacionalización» del punto $\mathrm{cl}$ (por ejemplo, la inscripción directa de un dominio punto $\mathrm{cl}$ desde cualquier parte del planeta, la existencia de agentes registradores extranjeros, etcétera). En paralelo, también era necesario poner atención a cambios que paulatinamente comenzaron a ser necesarios para actualizar el método local de resolución de controversias por nombres de dominio, proceso que fue favorecido por cambios legislativos que hicieron posible la construcción de una solución inédita en la manera de tramitar un arbitraje un nuestro país. ${ }^{19}$

Los ejes del cambio en materia de resolución de controversias, se pueden resumir en:

- Se estableció el procedimiento de revocación como el método general de disputa de un dominio punto cl, eliminándose las «solicitudes competitivas» como manera de disputar la solicitud de un nombre de dominio.

- La eliminación de la mediación como uno de los mecanismos de resolución de controversias. ${ }^{20}$

I9. Dentro de los cambios legislativos se pueden citar el reconocimiento del contrato electrónico (artículo 3 bis, b, de la Ley 19.496 sobre Protección de los Derechos de los Consumidores), la Ley I9.799 sobre Documentos Electrónicos, Firma Electrónica y Servicios de Certificación de Dicha Firma, las modificaciones al Código de Procedimiento Civil relativas al valor probatorio del instrumento electrónico, entre otros.

20. La justificación de este cambio se encuentra en que la mediación perdió progresivamente el impacto que tuvo a partir de la puesta en marcha en el año 2000. La asistencia 
- El reforzamiento de la cláusula compromisoria, fuente del arbitraje como método de resolución de controversias en dominios punto cl. $^{2 \mathrm{I}}$

- La Política de Resolución de Controversias es el procedimiento estándar de tramitación del arbitraje, y se entiende como el conjunto de reglas mínimas acordadas por todas las partes para el arbitraje y que el árbitro está obligado siempre a seguir en su procedimiento. ${ }^{22}$

- Los honorarios arbitrales tienen un monto determinado fijado a partir de reglas uniformes, junto a la forma de pago y a la constancia de su pago en el expediente electrónico. ${ }^{23}$

- El establecimiento de un procedimiento que se tramita en línea, concentrado, estructurado en base a los trámites de demanda, contestación, prueba y sentencia.

- La creación de un mecanismo procesal destinado a resguardar la independencia e imparcialidad del árbitro mediante un Comité de Evaluación de Árbitros, al cual también se le encarga el desarrollo

a la audiencia de mediación era voluntaria, y, al ser de este modo, la efectividad de la metodología se resintió notoriamente con la inasistencia creciente de uno o ambos competidores por el nombre. Se sumó a lo anterior que los competidores por una solicitud en trámite o los revocantes comenzaron a optar por el arbitraje, prescindiendo de llegar a acuerdos con los primeros solicitantes o titulares de los nombres, según hubiera sido el caso. Sin perjuicio de lo dicho, la mediación cumplió roles de alta significación que, si bien estaban alejados de su propósito esencial de obtener acuerdos que pusieran fin a las controversias, aportó a la difusión del sistema de nombres y sus mecanismos de resolución de controversias.

2I. Artículo 2 I de la Reglamentación de Dominios CL.

22. La existencia de un procedimiento uniforme era una demanda sentida por la comunidad. Tal como se expresó en otro lugar, por mucho que la práctica de los árbitros fuera igualándose en base a criterios más o menos parecidos, la determinación de las bases de procedimiento seguía siendo de resorte del árbitro. La Política se hizo cargo de estructurar un procedimiento único aceptado por árbitros y sus respectivas partes.

23. El pago del honorario por el arbitraje debe ser cubierto por quien solicita la revocación, de acuerdo a una tabla disponible en http://www.nic.cl/controversias/tabla_honorarios.html. Véase el párrafo 22 de la Política. La regulación de todos los aspectos inherentes al monto y forma de pago de los honorarios constituye un avance significativo en cuanto a asegurar a la comunidad bases ciertas y transparentes para su determinación. 
de criterios para las mejores prácticas del arbitraje y para la evaluación del plantel de árbitros. ${ }^{24}$

\section{EL PROCEDIMIENTO EN LÍNEA Y LA PLATAFORMA ELECTRÓNICA}

El procedimiento en línea está configurado en base a una plataforma electrónica que el Centro de Resolución de Controversias pone a disposición de los árbitros y las partes, y que consiste en un servicio dotado de una infraestructura tecnológica diseñada para permitir y facilitar la administración de los expedientes arbitrales.

La Política reafirma el rol que tiene NIC Chile y su Centro de Resolución de Controversias en relación a los procedimientos arbitrales, por cuanto su actuación en los expedientes se remite exclusivamente a ser proveedor del servicio de soporte tecnológico y no tiene ninguna facultad jurisdiccional u otra obligación que aquellas expresadas en la Política.

La plataforma de gestión de arbitrajes en línea pone a disposición de cada árbitro un sitio web con el estado de la tramitación de todos los expedientes que le han sido asignados. La identificación de cada árbitro está asegurada mediante un certificado digital que cada cual ha adquirido en cualquiera de las entidades prestadoras de servicios de certificación de firma electrónica acreditadas para operar de acuerdo a la legislación chilena. A su vez, las partes pueden acceder al expediente que corresponda al o los nombres de dominio en controversia a través de un sitio web donde disponen de diversas herramientas que les permiten interactuar con el tribunal, intervenir y participar en la tramitación del juicio arbitral. Para tener acceso al sitio web, el sistema provee a las partes de un mecanismo de claves de acceso, de uso exclusivo, siendo cada cual responsable de su administración, custodia, conservación o resguardo frente al uso indebido por terceros ajenos al juicio.

Todo el procedimiento arbitral consta en un expediente electrónico en el que se asientan e incorporan todas las actuaciones del árbitro con

24. El actual plantel de árbitros ha sido conformado en base al tercer concurso público convocado a comienzos del año 2013 el que amplió a 34 la dotación de abogados habilitados para resolver controversias. Los antecedentes del tercer concurso pueden consultarse en http://www.nic.cl/anuncios/20I30104-arbitros.html. 
expresión de la fecha y hora de realización. Asimismo, en él constan todas las comunicaciones a que dichas actuaciones dieren lugar y todos los documentos presentados por las partes, con expresión de la fecha y hora de recepción.

Para asegurar la unidad de la tramitación en el expediente electrónico, el árbitro, las partes y sus representantes no pueden realizar ninguna actuación de la cual no quede constancia en el expediente electrónico, y no tendrán ningún valor y se tendrán por no realizadas aquellas actuaciones que tengan lugar fuera de él o que no quede constancia en el mismo.

De acuerdo a un principio de confidencialidad relativa, durante la tramitación del procedimiento sólo el árbitro, las partes y sus representantes tienen pleno acceso a la consulta del expediente arbitral, el cual está disponible en línea permanentemente, excepto por interrupciones temporales causadas por requerimientos de mantención de servidores u otras de naturaleza similar. En este orden de ideas, durante el juicio arbitral, el árbitro, las partes y sus representantes deben mantener la confidencialidad de todas las pruebas aportadas al juicio y de todas las comunicaciones habidas entre ellas y el tribunal. Asimismo, bajo ninguna circunstancia alguno de ellos puede divulgar a terceros ajenos al juicio información cuyo conocimiento sea consecuencia de su acceso al expediente electrónico, sin perjuicio de la facultad del árbitro de entregar información del juicio a petición fundada de otro tribunal competente y de la publicación de la sentencia que resuelve el conflicto, la que tiene lugar luego de la correspondiente notificación. Una vez que se hubiera notificado el cierre del procedimiento arbitral, el expediente electrónico es público. Sólo por medio de una resolución que excepcionalmente así lo disponga, determinadas piezas del expediente pueden mantener el carácter de reservadas.

\section{DISEÑO DE LA PLATAFORMA DE GESTIÓN DE ARBITRAJES EN LÍNEA: LAS ETAPAS Y SUS USUARIOS}

\section{LAS ETAPAS}

La plataforma de gestión de arbitrajes en línea requiere la implementación del procedimiento de acuerdo a lo descrito anteriormente, considerando las siguientes etapas. 
a) Presentación de revocación: Funcionalidad que permite presentar una solicitud de revocación por un dominio inscrito en NIC Chile. Al presentar la solicitud de revocación, y una vez que conste el pago de la tarifa respectiva, el nombre de dominio pasa a tener un estado en conflicto, situación que es informada al titular. ${ }^{25}$

b) Crear expediente: Esta función permite crear el expediente electrónico en el sistema de arbitrajes en línea. Al momento de la creación del expediente, las partes del conflicto son notificadas y reciben la información necesaria para acceder al sistema y consultar el expediente correspondiente.

c) Tachas: En esta etapa las partes tienen la oportunidad de visualizar la nómina de árbitros vigentes en el sistema y pueden excluir a los que no desean considerar en la designación.

d) Designación: Es el momento en que tiene lugar el proceso de nombramiento del árbitro para el expediente, luego de concluida la etapa de tachas. Las partes y el árbitro son notificados de la designación.

e) Aceptación: El árbitro debe acceder al sistema y aceptar el nombramiento como árbitro del expediente correspondiente. Esta aceptación se realiza por medio de un documento firmado digitalmente que el árbitro debe informar al sistema.

f) Inhabilidad: Consiste en aquella etapa de la tramitación que permite a alguna de las partes promover en el expediente alguna cuestión que pueda afectar la imparcialidad e independencia del árbitro.

g) Consignación: En esta etapa el o los revocantes deben cumplir con el pago de los honorarios arbitrales.

h) Demanda: El o los revocantes deben presentar la demanda, dentro del plazo correspondiente, conjuntamente con los documentos probatorios que estime(n) convenientes.

i) Contestación: Proveída la demanda, el titular del nombre de dominio puede contestar la demanda, adjuntando los documentos probatorios que estime conveniente.

j) Sentencia: Concluidas las etapas de demanda y contestación, el juez árbitro debe dictar su sentencia. Para ello, informa al sistema el documento que contiene la sentencia donde podrá ordenar la asigna-

25. El ingreso de las solicitudes se efectúa en https://www.nic.cl/rcal/ingresoDominioRevocacion.do. 
ción del nombre de dominio al demandante o que se mantenga la actual asignación.

k) Ejecución de sentencia: El Centro de Resolución de Controversias ejecuta la sentencia cuyo cumplimiento le ha ordenado el árbitro.

1) Publicación de la sentencia: Inmediatamente de cumplido lo ordenado por el árbitro, la sentencia queda disponible para su consulta en un sitio de acceso público dispuesto especialmente para tales efectos. ${ }^{26}$

\section{LOS USUARIOS}

El procedimiento electrónico requiere de distintos tipos de usuarios con permisos acordes a la etapa en curso del expediente. Los usuarios identificados para este sistema son los siguientes.

a) Revocante: Usuario que presenta la solicitud de revocación por un nombre de dominio en el Centro de Resolución de Controversias. Posteriormente, en la tramitación del expediente electrónico, en el sistema de arbitrajes en línea, cumplirá con las funciones correspondientes al demandante, si es que así corresponde. Se debe considerar que puede existir más de un revocante en un mismo expediente.

b) Titular: Corresponde al asignatario del nombre de dominio contra el cual se presentó una solicitud de revocación. Posteriormente, en la tramitación del expediente electrónico podrá cumplir con las funciones de demandado, dependiendo si se han dado las condiciones previas en el procedimiento.

c) Árbitro: Es el usuario que cumple el rol de juez en la tramitación del expediente electrónico, condición que adquiere efectivamente una vez que aceptó el arbitraje.

d) Administrador: Corresponde al usuario gestor del sistema, que coordina el funcionamiento de las funcionalidades de la plataforma, resolviendo incidentes, consultas de árbitros y partes, etcétera.

\section{DIAGRAMA}

En el diagrama I se presenta una secuencia de las principales etapas del procedimiento y las principales relaciones con los usuarios.

26. Véase http://www.nic.cl/rcal/fallos.do. 


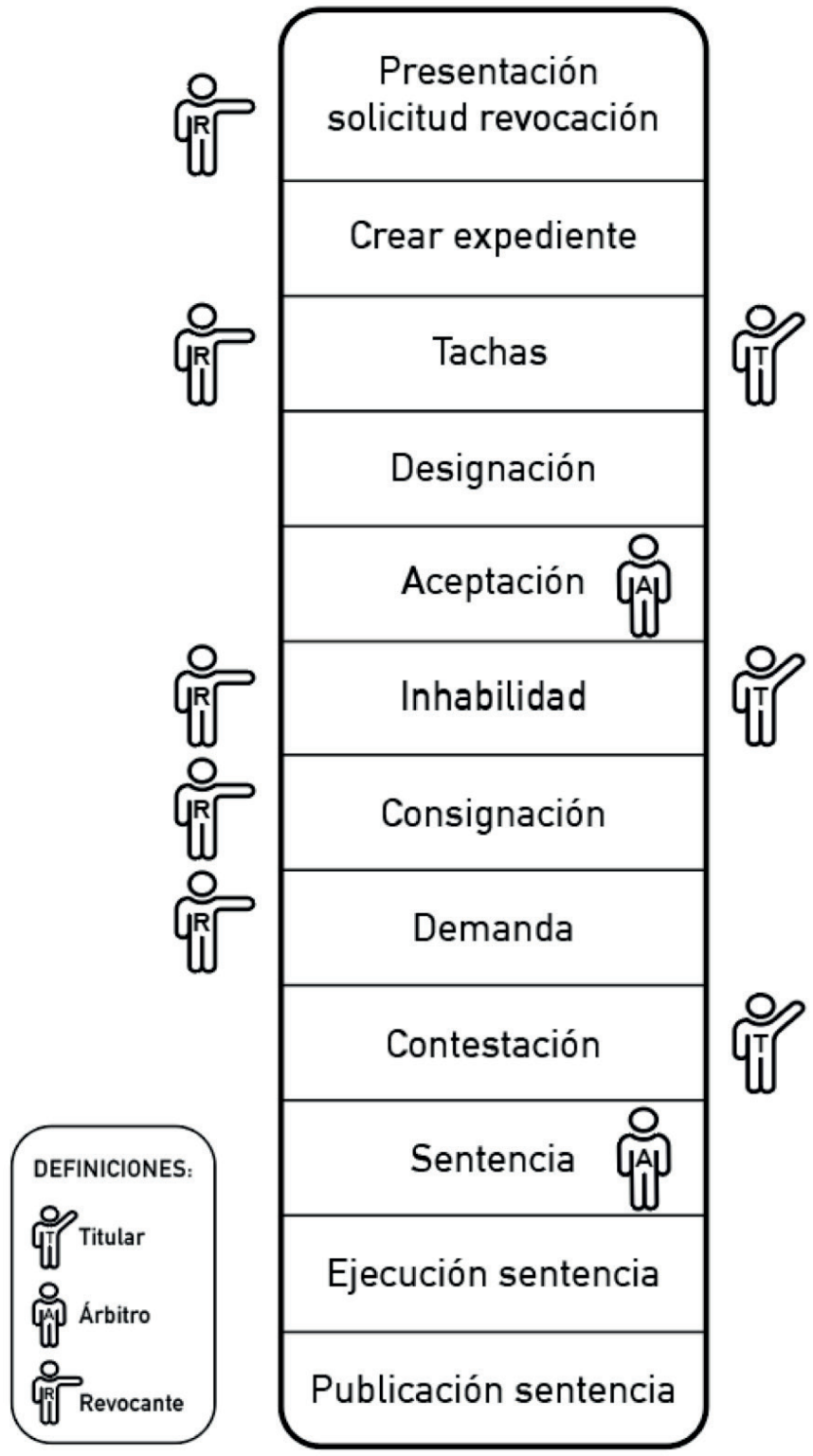

Diagrama 1. Etapas del procedimiento en línea. 


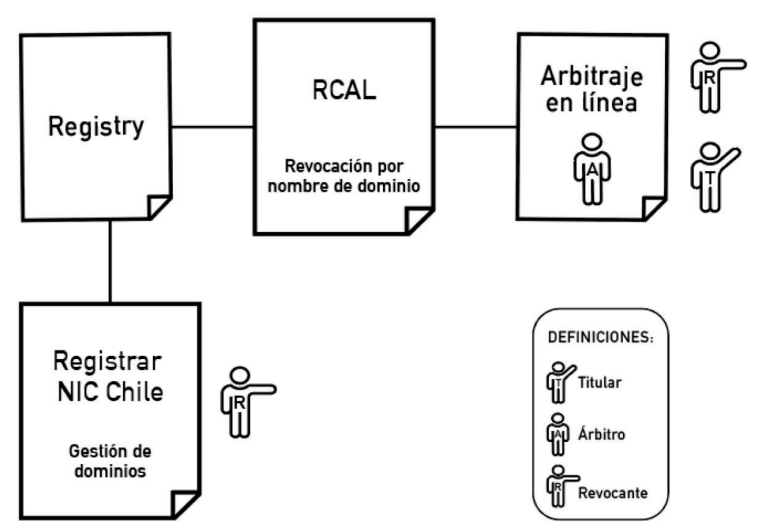

Diagrama 2. Arquitectura de los sistemas.

\section{ESTRUCTURA DE LOS SISTEMAS}

Con la entrada en vigencia de las nuevas políticas de dominios punto cl en diciembre del 20I3, comenzó a operar una nueva arquitectura de sistemas que implementan las funciones definidas en las nuevas políticas y procedimientos. El sistema de arbitrajes en línea forma parte de esta nueva arquitectura, representada en el diagrama 2.

Las principales responsabilidades de los sistemas en esta nueva arquitectura son:

- Registry: Mantener el registro centralizado de los dominios punto cl y permitir la existencia y funcionamiento técnico de los dominios inscritos en Internet. Este sistema recibe operaciones sobre nombres de dominio desde el sistema Registrar y el sistema de Resolución de Controversias y Arbitraje en Línea (RCAL).

- Registrar NIC Chile: Provee las funciones necesarias para que los usuarios puedan inscribir y gestionar los nombres de dominio. Esto considera las operaciones de inscripción, renovación, modificación, eliminación, restauración y gestión de contactos, entre otras. Las operaciones se realizan por medio de una cuenta de usuario y son comunicadas al sistema Registry cuando corresponde.

- Resolución de Controversias y Arbitrajes en Línea (RCAL): En este sistema se gestionan las solicitudes de revocación que presentan los usuarios para un nombre de dominio, ordena la creación 
de los expedientes electrónicos en el sistema de arbitraje en línea y también se gestiona la ejecución y publicación de las sentencias arbitrales. Al ejecutar las sentencias, informa al sistema Registry de los cambios de estado en el dominio inscrito.

- Arbitraje en Línea: Sistema responsable de la tramitación de un expediente electrónico. Permite que las partes y el árbitro desarrollen las actividades procesales que corresponde a cada uno de sus roles en el juicio. Finalmente, informa al sistema RCAL las sentencias arbitrales para su ejecución y publicación.

El sistema de arbitraje en línea considera accesos independientes por rol de usuario en el sistema. En la práctica, los árbitros acceden por una URL diferente a la que usan las partes. Por otro lado, para la presentación gráfica de las interfaces del sistema se consideraron aspectos de usabilidad de sistemas, con el fin de facilitar el uso y guiar a los usuarios de la mejor forma en las acciones que deben realizar.

Las tecnologías de desarrollo del sistema de arbitraje en línea están alineadas con las usadas en los nuevos sistemas de NIC Chile y son parte del estándar de la industria para este tipo de sistemas que operan en plataforma web. De esta forma, se logra disminuir los requerimientos de acceso para los usuarios y se permite operar el sistema desde prácticamente cualquier navegador de internet.

Finalmente, considerando los sistemas RCAL y Arbitrajes en Línea, se escribieron más de 45.000 líneas de código, y desde la puesta en marcha se han generado más de I 80 nuevas versiones de estos sistemas, reflejando las constantes mejoras y nuevas funcionalidades que se han agregado.

\section{ESTADÍSTICAS DEL SISTEMA}

Desde el inicio de su funcionamiento efectivo en junio de 20I4, el sistema ha permitido y facilitado la sustanciación remota de los conflictos en expediente digitales. La experiencia desarrollada a la fecha permite concluir que la puesta en operaciones de un servicio de esta naturaleza ha resultado exitosa, particularmente en lo relativo a permitir que árbitros y litigantes puedan ejercer eficientemente sus actividades y derechos en un ambiente totalmente digitalizado. 


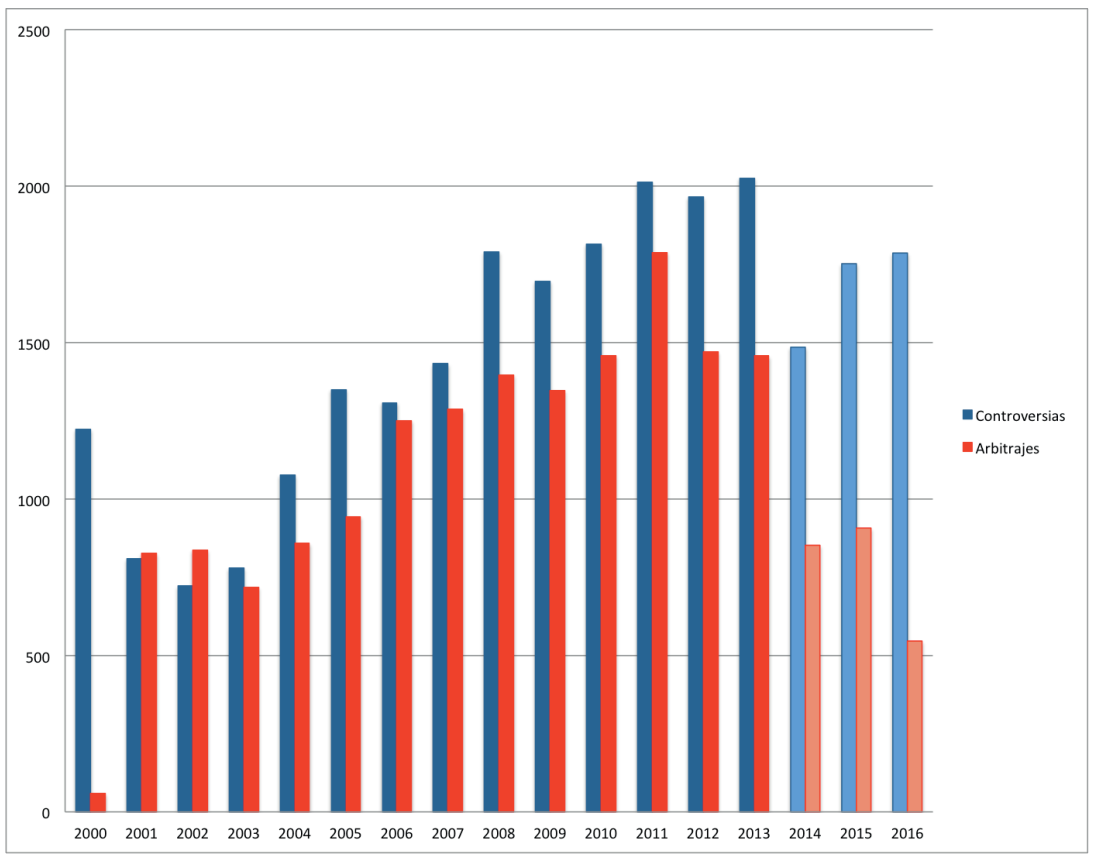

Gráfico 1. Cantidad de controversias y de arbitrajes.

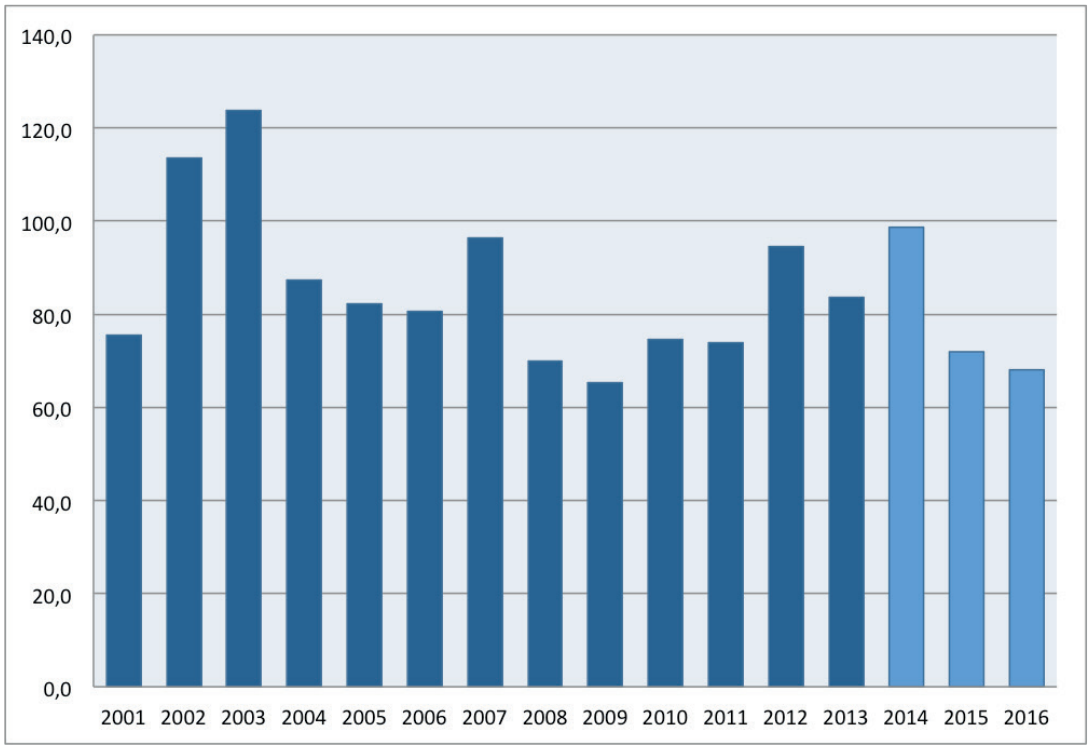

Gráfico 2. Tiempo promedio duración de los arbitrajes. 
A continuación se presenta algunos indicadores del funcionamiento del sistema desde sus inicios hasta la actualidad. Los tres últimos años corresponden al nuevo sistema en línea. El gráfico I muestra el número de controversias planteadas y las compara con las que efectivamente llegaron a arbitraje. El gráfico 2 muestra el tiempo promedio de duración de los arbitrajes.

\section{MEJORAS}

Desde el inicio de las operaciones del procedimiento en línea, la preocupación fundamental de la administración de las controversias fue dotar al servicio de la suficiente estabilidad y proveer de un manejo eficiente de los incidentes que pudieron haber ocurrido. En cerca de tres años de funcionamiento, el servicio ha sido una herramienta plenamente operativa y eficaz para el cumplimiento de sus objetivos. Sin perjuicio de lo expresado, se ha detectado la pertinencia de introducir mejoramientos en determinados aspectos de la gestión, dentro de los cuales se puede mencionar:

- Mejorar las herramientas de gestión de los expedientes, diferenciadas por tipos de usuarios.

- Promover la litigación efectiva de los titulares de nombres de dominio en revocación. Para esto se ha convocado a las clínicas jurídicas de diversas universidades, de manera de facilitar a los titulares de dominios en revocación el acceso a asesoría legal gratuita, si así lo desean.

- Revisar los criterios de usabilidad de las diversas herramientas del sistema y suministro de información a los usuarios en el expediente electrónico.

\section{REFERENCIAS}

Carey, Jorge y Gonzalo Fernández (2007). «Los costos del arbitraje». En Estudios de Arbitraje. Libro homenaje al profesor Patricio Aylwin Azócar (pp. 2I I-235). Santiago: Jurídica.

Piquer, José M. y Patricio V. Poblete (r999). «Domain Name Conflict Resolution Under the .CL Top-Level Domain», ISOC'99 Con- 
ference, San Jose, USA. Disponible en https://www.isoc.org/inet99/ proceedings $/ 3 \mathrm{~h} / 3 \mathrm{~h} \_2 . \mathrm{htm}$.

Poblete, Patricio V. (20I6). «Histórica Reunión de ICANN en Marrakech». BITS de Ciencia, I 4: 20-24.

VAldés, Margarita (2016). «Un día histórico para Internet», El Mercurio, 3 de octubre de 2016 , p. A2, disponible en http://www.elmercurio.com/blogs/20 I 6/Io/o3/4549 I/Un-momento-historico-para-internet.aspx.

\section{SOBRE LOS AUTORES}

Luis Arancibia Medina es abogado. Licenciado en Ciencias Jurídicas y Sociales de la Universidad de Zaragoza, España. Coordinador del Centro de Resolución de Controversias de NIC Chile, Universidad de Chile. Su correo electrónico es lam@nic.cl.

Patricio V. Poblete es Ingeniero Matemático de la Universidad de Chile. Doctor en Ciencias de la Computación de la Universidad de Waterloo, Canada. Profesor Titular de la Facultad de Ciencias Físicas y Matemáticas de la Universidad de Chile y Director de NIC Chile. Su correo electrónico es ppoblete@nic.cl.

José Urzúa Reinoso es Ingeniero Civil en Computación de la Universidad de Chile y Magíster en Ciencias mención Computación de la misma Universidad. Jefe de Desarrollo de Sistemas en NIC Chile y Profesor jornada parcial del Departamento de Ciencias de la Computación de la Facultad de Ciencias Físicas y Matemáticas de la Universidad de Chile. Su correo electrónico es jose@nic.cl.

Margarita Valdés Cortés es abogada, egresada de la Universidad de Chile. Directora Legal y Comercial de NIC Chile, Universidad de Chile. Profesora invitada en el Magister de Nuevas Tecnologías de la misma Universidad. Magíster en Gestión de Negocios de la Universidad Adolfo Ibáñez.Su correo electrónico es mvaldes@nic.cl.

Este trabajo fue recibido el I 6 de noviembre y aprobado el 6 de diciembre de 2016 . 